\title{
Hydrogen bonding interactions of benzylidene type Schiff bases studied by vibrational spectroscopic and computational methods
}

\author{
Ottó Berkesi, ${ }^{a}$ Tamás Körtvélyesi, ${ }^{a}$ Csaba Hetényi, ${ }^{b}$ Tamás Németh ${ }^{a}$ and István Pálinkóo ${ }^{* c}$
}

\author{
${ }^{a}$ Department of Physical Chemistry, University of Szeged, Szeged, Rerrich B. tér 1, \\ H-6720 Szeged, Hungary \\ ${ }^{b}$ Department of Medical Chemistry, University of Szeged, Szeged, Dóm tér 8, H-6720 Szeged, \\ Hungary \\ ${ }^{c}$ Department of Organic Chemistry, University of Szeged, Szeged, Dóm tér 8, H-6720 Szeged, \\ Hungary.E-mail: palinko@chem.u-szeged.hu; Fax: +36 62544 200; Tel: +36 544288
}

Received 27th January 2003, Accepted 28th March 2003

First published as an Advance Article on the web 9th April 2003

The structural features of four benzylidene type Schiff bases [(E)-benzaldehyde- $N$-phenyl imine, (A) (E)-2-hydroxybenzaldehyde- $N$-phenyl imine (B) $(E)$-benzaldehyde- $N$-2-hydroxyphenyl imine (C) (E)-2-hydroxybenzaldehyde- $N$-2-hydroxyphenyl imine (D)] were studied by FT-IR spectroscopy in solution, photoacoustic and Raman spectroscopies in the solid state and quantum chemical calculations. It was found that molecule $\mathbf{D}$ dimerised in the solid state with concomitant loss of aromaticity in the benzylidene ring. Beside the intermolecular $\mathrm{C}=\mathrm{O} \cdots \mathrm{HO}$ hydrogen bonds, intramolecular $\mathrm{N}-\mathrm{H} \cdots \mathrm{C}=\mathrm{O}$ hydrogen bonds could be found experimentally as well as computationally. Spectra taken in solution and ab initio quantum chemical calculation helped to identify hydrogen bonding interactions occurring for compounds $\mathbf{B}$ and $\mathbf{C}$. Intramolecular $\mathrm{OH} \cdots \mathrm{N}$ hydrogen bond predominated in molecule B, while this interaction, although it existed, was weaker.

\section{Introduction}

Schiff bases in general are important molecules from several aspects. Imines are crucial synthetic intermediates ${ }^{1}$ (e.g., their hydrogenation leads to amines, they undergo radical dimerisation, etc.), they do have biological $\operatorname{role}^{2}(e . g$. , in vision, in determining the flexibility of the wall of the veins, etc.) and the salicylidene types may exibit thermocromic ${ }^{3}$ or photocro$\mathrm{mic}^{4}$ properties. These latter two are connected to structural features, i.e., to the possibility of proton transfer complex formation. No wonder that the benzylidene type Schiff bases are widely studied molecules, however, those containing $\mathrm{OH}$ groups in both ring are concerned by relatively few studies. ${ }^{5-8}$ This is somewhat surprising, since the presence of two $\mathrm{OH}$ groups provides rich possibility for hydrogen bonding interactions that may greatly influence the structure of the compounds. If the $\mathrm{OH}$ groups are in appropriate positions relative to the $\mathrm{C}=\mathrm{N}$ functionality, both intramolecular and intermolecular $\mathrm{H}$-bonds may appear.

In this work various benzylidene type Schiff bases without (base compound) and with one or two hydroxy groups were the subjects of studying both kinds of interaction in the solid state. Photoacoustic spectroscopy (PAS) and Raman spectroscopy were used as primary experimental tools. Molecular modeling was also applied to help visualising and rationalising experimental results obtained.

\section{Experimental}

The aggregation properties of four Schiff bases were investigated (Fig. 1). The base compound was $(E)$ - $N$-benzylidene aniline [(E)-benzaldehyde- $N$-phenyl imine, (A)] prepared by the condensation of benzaldehyde (Aldrich) and aniline (Aldrich) following the recipe given in ref. 7 . Three of its derivatives were also synthesized by the same method. They were as follows: (E)-2-hydroxybenzaldehyde- $N$-phenyl imine (B), $(E)$-benzaldehyde- $N$-2-hydroxyphenyl imine $(\mathbf{C})$ and $(E)$-2-hydroxybenzaldehyde- $N$-2-hydroxyphenyl imine (D).

Infrared spectra were recorded on a Bio-Rad Digilab Division FTS-65A/896 Fourier Transform Infrared (FTIR) spectrometer. Photoacoustic IR spectra (PAS) of solid samples were registered using an MTEC-200 detector. The absorbance spectra were recorded either in Nuyol or in Fluorolube mulls in the range of $4000-400 \mathrm{~cm}^{-1}$ using a liquid nitrogen cooled MCT (Mercury-Cadmium-Telluride) detector. Spectra of every compound were also recorded in acetone- $\mathrm{d}_{6}$, compound D in tetrahydrofuran (thf), chloroform (chf) and carbon tetrachloride (ctc). The concentration of the compounds was 0.01 mol dm${ }^{-3}$, to minimise the chance of aggregation. The optical length of the cell was $0.1 \mathrm{~mm}$. The optical resolution was 2 $\mathrm{cm}^{-1}$ and 256 scans were collected for each spectrum.

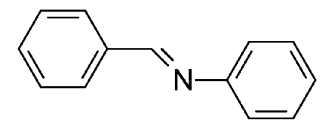

$\mathbf{A}$

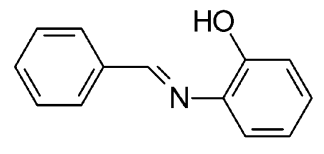

C

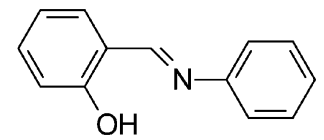

B

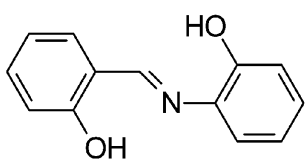

D
Fig. 1 The molecules studied.

Phys. Chem. Chem. Phys., 2003, 5, 2009-2014

DOI: $10.1039 / \mathrm{b} 301107 \mathrm{k}$

This journal is (C) The Owner Societies 2003 
Raman spectra were taken on a dedicated Bio-Rad Digilab Division Fourier Transform Raman spectrometer using the $1064 \mathrm{~nm}$ line of a T-10-106c type Nd:YVO 4 laser from Spectra Physics. The laser power at the sample position was $500 \mathrm{~mW}$. The spectra of the solid samples were recorded in a $5 \mathrm{~mm}$ NMR tube with $4 \mathrm{~cm}^{-1}$ optical resolution and accumulating 128 scans.

\section{Computational methods}

Full geometry optimisation of the molecules were performed by semiempirical and ab initio quantum chemical routines included in the Hyperchem 7.0 package running on a PC. ${ }^{9}$ Structures were preoptimised by the PM3 method, ${ }^{10}$ then the optimum geometries were taken as the inputs for $\mathrm{HF} / 6$ $31 \mathrm{G}^{*} / / \mathrm{HF} / 6-31 \mathrm{G}^{*}$ ab initio calculations. The fully optimised geometries had positive definite force matrices with both methods indicating that minima were found indeed.

\section{Results and discussion}

\subsection{General considerations}

There are two types of functional groups in the molecules to take into consideration when association properties are concerned. One is the hydroxy group and the other is the lone pair of the $\mathrm{C}=\mathrm{N}$ group. The oxygen of the hydroxy group is able to play a role as the hydrogen donor atom in a hydrogen bond and the nitrogen (with the lone electron pair) can be the acceptor.

Band predominated by the stretching vibrations of the hydroxy group $\left(\nu_{\mathrm{OH}}\right)$ can be found in the higher wavenumber region of the mid-IR spectrum due to the low reduced mass of the $\mathrm{OH}$ group. It is well-known that the band is very sensitive to changes in the chemical environment. It is broad and moves towards lower wavenumbers when the group is in hydrogen bonding interaction and sharp and appears at higher wavenumbers when such interaction does not exist.

In the solid state the materials are crystalline. The crystal structure may be kept together by various forces. In molecular crystals, this is the case with our model compounds, they are hydrogen bonds, van der Waals interactions and many other still not fully explored forces (e.g., $\mathrm{C}-\mathrm{H} \cdots \pi, \pi \cdots \pi$, etc.). Undoubtedly, the most important ones are the hydrogen bonds (they are strong and directional), thus, if they can exist, they will have central role. In solution it is possible to disrupt the intermolecular hydrogen bonding network of the molecules, for instance, by dilution. Thus, comparing the vibrational spectra of an appropriately diluted solution and that of the solid state may provide information on the nature of the hydrogen bonding pattern (whether intermolecular hydrogen bonds are present or not) prevailing in the molecular crystal.

Among our models molecule D contains two $\mathrm{OH}$ groups, consequently, the largest variety of hydrogen bondings are expected here. In the following sections we shall focus on methods giving direct information about hydrogen bonding of these molecules in the solid state.

\subsection{Structural considerations based on vibrational spectroscopic measurements in the solid state}

The photoacoustic spectra taken in the solid phase, in the range of the $\nu_{\mathrm{OH}}$ vibrations, are shown for the studied molecules in Fig. 2.

For the non-substituted molecule (A) only the bands of the aromatic $\mathrm{C}-\mathrm{H}$ stretching vibrations were found, while the other three compounds featured very diverse spectral properties. The molecules containing a hydroxy group in ortho posi-

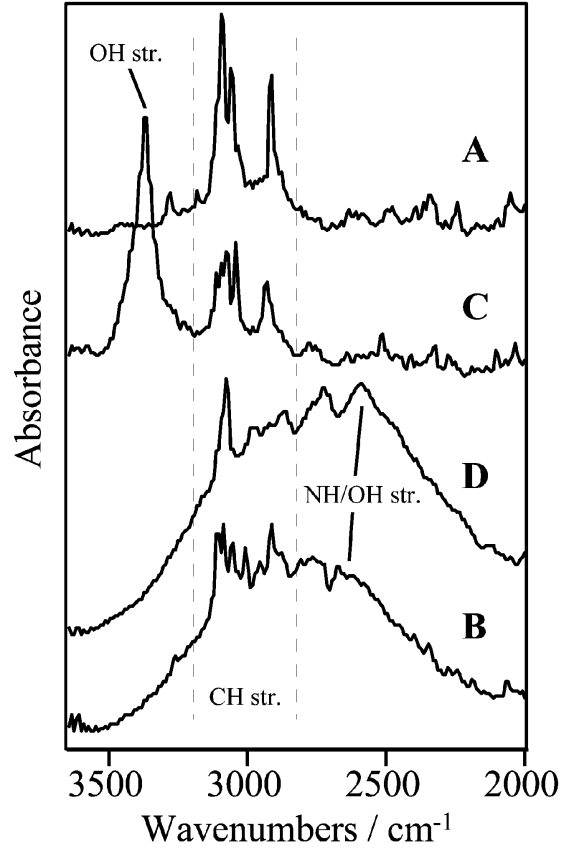

Fig. 2 PAS-IR spectra of compounds A-D in the solid state in the range of hydroxyl stretching vibrations.

tion in the ring connected to the carbon atom of the $\mathrm{C}=\mathrm{N}$ group (B and $\mathbf{D}$ ) had broad bands shifted enormously to low wavenumbers, indicating strong hydrogen bond involving the hydroxy group. On the other hand, molecule $\mathbf{C}$, in which the hydroxy group was located in the ortho position in the ring connected to the nitrogen atom of the $\mathrm{C}=\mathrm{N}$ group, showed a relatively narrow band, only somewhat below the region of the isolated $\mathrm{OH}$ bands, indicating that the association was weaker in this molecule. However, such a band was missing from the spectrum of compound $\mathbf{D}$, in spite of the fact that it also contained an ortho hydroxy group in the ring connected to the nitrogen atom of the $\mathrm{C}=\mathrm{N}$ group. The $\mathrm{OH}$ band was shifted to lower wavenumbers like that of compound $\mathbf{B}$, indicating hydrogen bonding that involves the $\mathrm{OH}$ group.

Those normal modes that were predominated by the other important functional group in the molecule were possible to identify because of the relatively high force constant of the carbon-nitrogen double bond (Fig. 3).

The spectra of three samples (A, B and C) featured largely the same type of bands in the range of $1650-1550 \mathrm{~cm}^{-1}$. Bands at the highest wavenumbers were dominated by the $\mathrm{C}=\mathrm{N}$ stretching coordinate, while the bands at the lower wavenumbers were assigned to the skeletal modes of the rings. The shift of the $\mathrm{C}=\mathrm{N}$ stretching mode to lower wavenumbers may be attributed to the association occuring between the $\mathrm{OH}$ and $\mathrm{C}=\mathrm{N}$ groups. ${ }^{11,12}$ The shift was most pronounced for compound $\mathbf{B}$, where the formation of intramolecular hydrogen bond is well-known and studied. ${ }^{3,5,8,13-19}$ Compound $\mathbf{C}$ displayed smaller shift, in accordance with the conclusion drawn from the $\mathrm{OH}$ stretching region, i.e., the association between the $\mathrm{OH}$ and $\mathrm{C}=\mathrm{N}$ groups is much weaker in this molecule.

The spectral features of compound $\mathbf{D}$ were significantly different from those of the other molecules. The band at 1636 $\mathrm{cm}^{-1}$ is too high to be acceptable as $\mathrm{C}=\mathrm{N}$ stretching mode, rather, it can be assigned to $\mathrm{C}=\mathrm{O}$ stretching vibration. Further intriguing observation was the missing of a band in the 1580 $1560 \mathrm{~cm}^{-1}$ range. In the other molecules this band could be assigned to the skeletal mode of the aromatic ring connected to the $\mathrm{C}$ atom of the $\mathrm{C}=\mathrm{N}$ group. ${ }^{11,12}$ Its lack in molecule $\mathbf{D}$ may indicate the possible destruction of the benzoic structure of the ring. The strong doublet around $1537 \mathrm{~cm}^{-1}$ was also unexpected. 


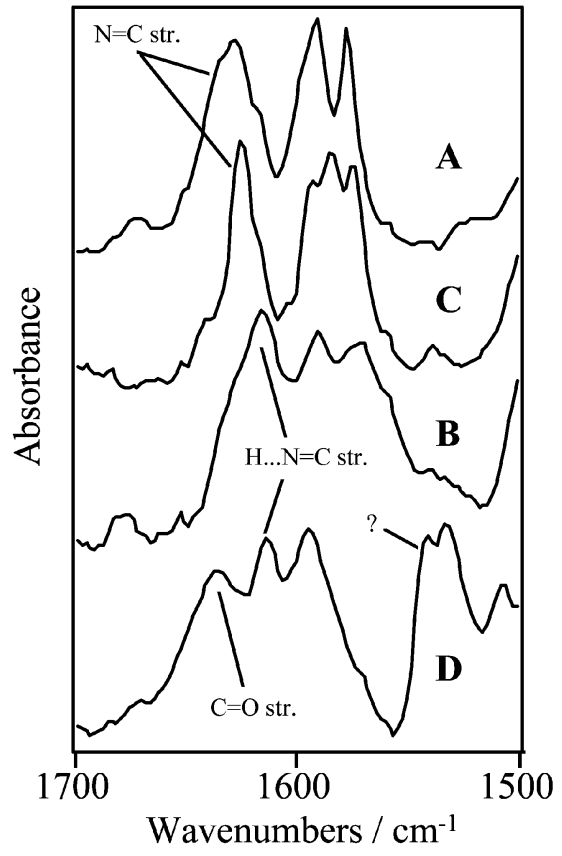

Fig. 3 PAS-IR spectra of compounds A-D in the solid state in the range of $\mathrm{C}-\mathrm{C}$ double bond stretching vibrations.

Most of the above-mentioned spectral features was observed by Ledbetter ${ }^{8}$ in the solid-state spectrum of molecule $\mathbf{B}$, but he found a strong band at $1627 \mathrm{~cm}^{-1}$. The presence of both tautomeric forms in the solid sample was predicted on the basis of that band. However, in our hands the spectrum of molecule $\mathbf{B}$ has never shown a band at that wavenumber, thus, it was suspected that the spectrum of either our or his sample had been influenced by the method of sample preparation. Since we used photoacoustic spectroscopy, when no physical treatment of the sample preceeded the measurement, we are certain that no experimental artifacts influenced our results. Furthermore, the spectra recorded in Nuyol and the Fluorolube mull also lacked the band in question, thus, the $\mathrm{KBr}$ pellet technique must have been responsible for the alteration. Consequently, only spectrum $\mathbf{D}$ indicated structural changes. It was assumed that structural rearrangement, the loss of aromaticity in the ring connected to the nitrogen took place.

The comparison of the Raman and IR-PAS spectra for the molecules confirmed this assumption, since higher symmetry element was only found for compound $\mathbf{D}$. The double bond region, displayed in Fig. 4, indicated the presence of an inversion centre in the structure.

The pair of the band at $1636 \mathrm{~cm}^{-1}$ in the PAS-IR spectrum was found at $1625 \mathrm{~cm}^{-1}$ in the Raman spectrum. Deconvolution of both spectra revealed another pair of bands, which showed the exclusion rule at $1614 \mathrm{~cm}^{-1}$ in the PAS-IR and at $1610 \mathrm{~cm}^{-1}$ in the Raman spectrum. Three pairs of bands in the aromatic $\mathrm{C}-\mathrm{C}$ skeletal region appeared at the same wavenumbers showing that the interaction between the rings was too small or the central symmetry applies only for part of the system. An X-ray diffraction study of compound D showed polymeric chain structure, where the molecular chains were formed through hydrogen bonds and four molecules, belonging to two different chains were in the unit cell showing central symmetry in the crystallographic coordinate system. ${ }^{6}$ The geometric parameters extracted from the X-ray data suggested an intermediate structure between the benzoic and the quinoidal form at the ring connected to the $\mathrm{C}$ atom of the $\mathrm{C}=\mathrm{N}$ bond.

It would have been easy to accept that the structure and the spectral features were in agreement, but we think it is fair to note that the assignments of the new $\mathrm{C}=\mathrm{O}$ stretching mode

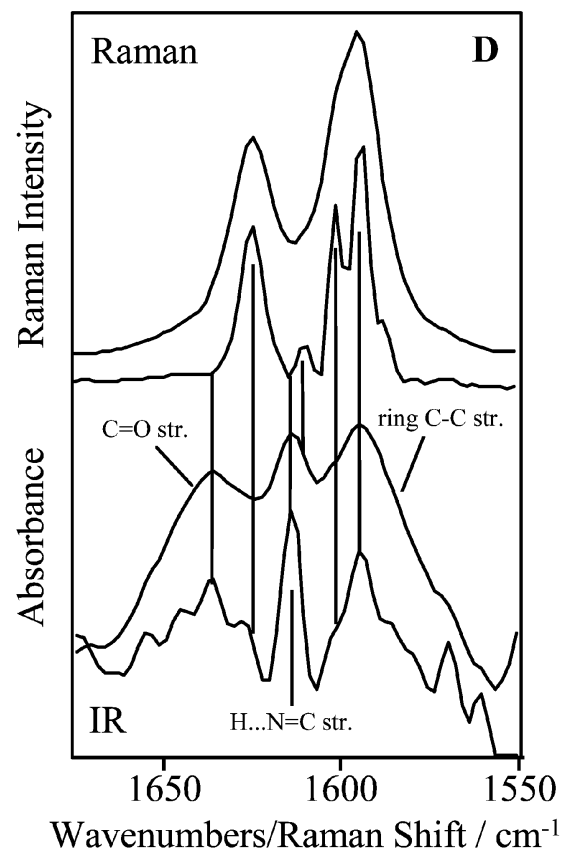

Fig. 4 Deconvolution of the PAS-IR and the Raman spectra of compound $\mathbf{D}$ indicating central symmetry in the structure.

and the unperturbed $\mathrm{C}=\mathrm{N}$ band are somewhat disturbing. There is no explanation concerning the low intensity of the Raman band at $1610 \mathrm{~cm}^{-1}$ either.

\subsection{Structural considerations based on vibrational spectroscopic measurements in solution}

The spectrum of compound $\mathbf{D}$ was simplified on dissolution. The $\mathrm{OH}$ stretching region of the spectra recorded in deuteroacetone (Fig. 5) showed similarities between compounds A and $\mathbf{B}$ and compounds $\mathbf{C}$ and $\mathbf{D}$. Spectra for each molecule resembled each other in the double bond region.

For compound $\mathbf{D}$ the $\mathrm{C}=\mathrm{N}$ stretcing band was found at 1619 $\mathrm{cm}^{-1}$, the band assigned as $\mathrm{C}=\mathrm{O}$ stretching mode disappeared

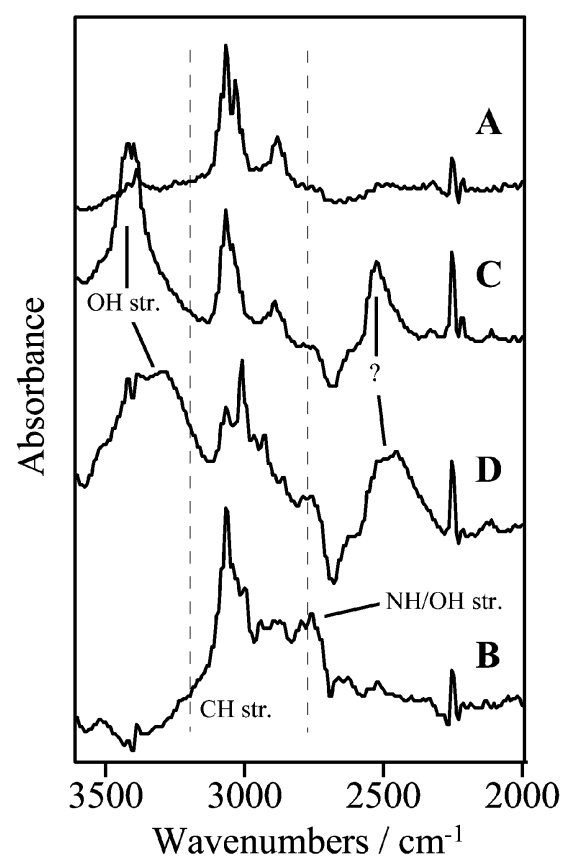

Fig. 5 FT-IR spectra of compounds A-D dissolved in acetone- $\mathrm{d}_{6}$ in the range of hydroxyl stretching vibrations. 


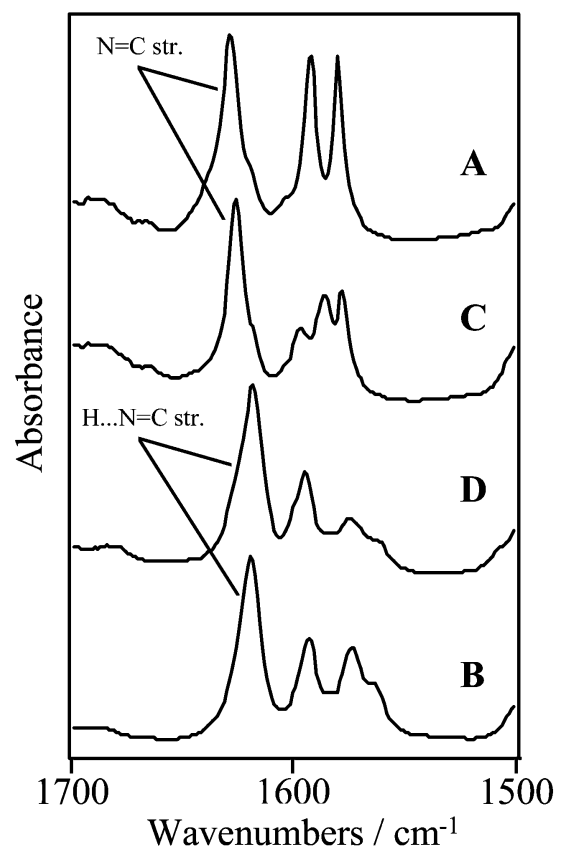

Fig. 6 FT-IR spectra of compounds A-D dissolved in acetone-d ${ }_{6}$ in the range of $\mathrm{C}-\mathrm{C}$ double bond stretching vibrations.

and the missing aromatic $\mathrm{C}-\mathrm{C}$ skeletal bands reappeared in the range of $1580-1560 \mathrm{~cm}^{-1}$. Spectra of compounds $\mathbf{B}$ and $\mathbf{D}$ became nearly identical between 1700 and $1500 \mathrm{~cm}^{-1}$ indicating the same type of association (Fig. 6).

The nature of the solvent also influenced the association. Solvents with low ability to associate with the solute reveal the real nature of the solute as indicated by the spectra recorded in chloroform and carbon tetrachloride displayed in Fig. 7 for molecule $\mathbf{D}$.

The $\mathrm{OH}$ stretching region (Fig. 7(a)) showed bands at around $3425 \mathrm{~cm}^{-1}$, typical for $\mathrm{OH}$ group in intramolecular hydrogen bonding. The broad band occurring as a consequence of intermolecular association could not be found. The double bond region (Fig. 7(b)) also verified that chlorinated solvents reduced interactions. The $\mathrm{C}=\mathrm{N}$ stretching bands narrowed considerably and shifted slightly to higher wavenumbers indicating that the rate of exchange reactions decreased. The relative intensities and the positions of the bands assigned to the ring skeletal modes were also altered. The alteration of the relative orientation of the rings and the bridging $\mathrm{C}=\mathrm{N}$ group could influence those bands. The more co-planar the molecule the higher the possibility of the contribution of the $\mathrm{C}=\mathrm{N}$ stretching coordinate to the modes dominated by skeletal vibrations. An intramolecular hydrogen bridge forces the $\mathrm{C}=\mathrm{N}$ group into the plane of the ring connected to the $\mathrm{C}$ atom.

\subsection{Conclusions from vibrational spectroscopic measurements and further expectations from quatum chemical calculations}

Experiments revealed that in the solid state dimerisation was typical for molecule D. It should have occurred through the association of the quinoidal tautomeric form. The broad and drasticaly downward shifted $\mathrm{OH} / \mathrm{NH}$ bands indicated intramolecular hydrogen bonds as well, just like in molecule B. Molecule $\mathbf{C}$ did not seem to be dimerised, however, intramolecular hydrogen bonding similar to molecule $\mathbf{B}$, albeit significantly weaker, could be identified. Dissolution of the compounds in deuteroacetone and other solvents broke up the dimer of molecule $\mathbf{D}$ and the quinoidal structure ceased to exist. Features resembling both molecules $\mathbf{B}$ and $\mathbf{C}$ could be observed in solution, i.e., signs of intramolecular hydrogen bonds.

The qualitative picture provided by vibrational spectroscopies can be made more quantitative by quantum chemical calculations. An $a b$ initio treatment applying a relatively large basis set may give close to reliable structures and geometric data. Among geometric data those of the inter- and intramolecular hydrogen bonds are important. Calculations are also expected to provide a fair estimate of hydrogen bond strength, and hence the gains in energy due to their formation.
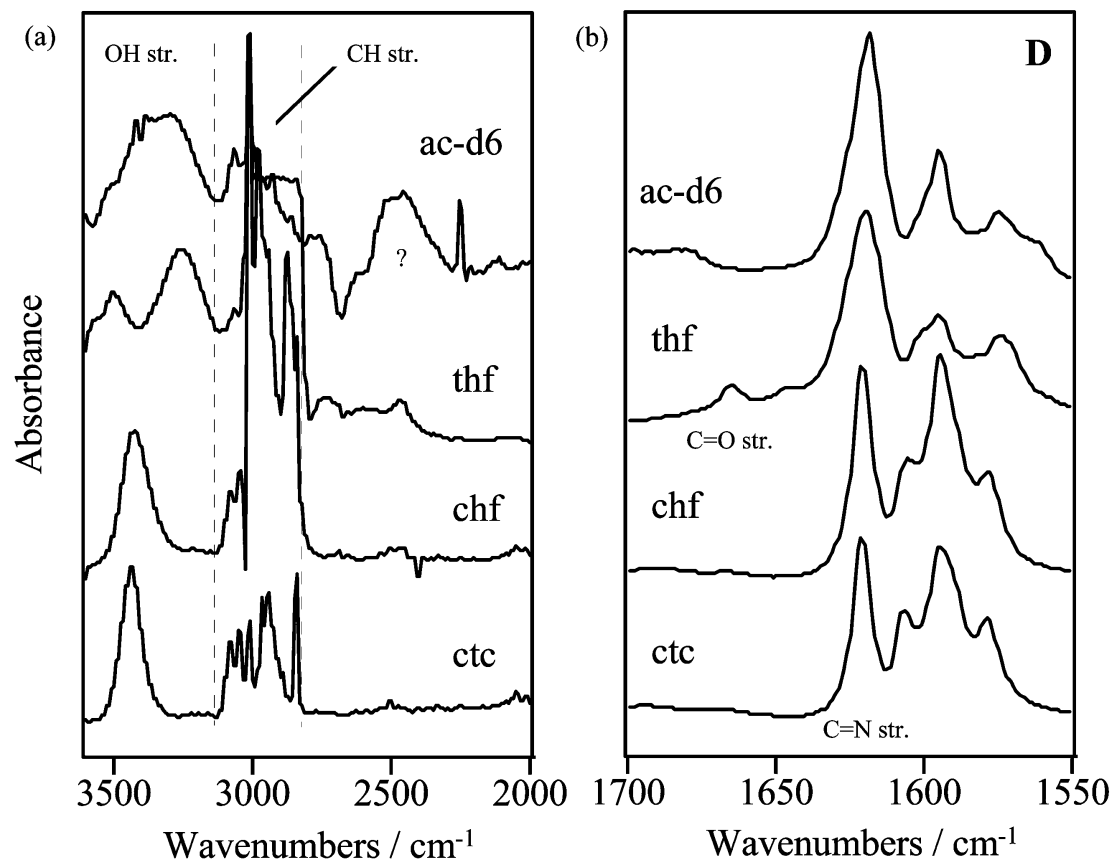

Fig. 7 FT-IR spectra of compound $\mathbf{D}$ dissolved in acetone- $\mathrm{d}_{6}$, tetrahydrofurane (thf), chloroform (chf) or carbon tetrachloride (ctc) in the range of (a) hydroxyl and (b) C-C stretching vibrations. 


\subsection{Structural considerations based on molecular modeling}

For molecular modeling two conformer pairs of molecules $\mathbf{B}$ and $\mathbf{C}$ and four of molecule $\mathbf{D}$ were used as starting compounds. In the starting conformations of these monomers (modeling the molecules in solution and, except for molecule D, in the solid state as well) the hydrogen of the $\mathrm{OH}$ groups were either directed towards the $\mathrm{N}$ atom in the molecules ("in" conformation) or away from it ("out" conformation). For molecule D containing two $\mathrm{OH}$ groups this meant four possibilities. It was expected that hydrogen bonding interactions lower the total energies of the molecules, thus, the energy gain was calculated relative to the total energies of the (all) "out" conformers. (For the PM3 semiempirical calculations the formation enthalpies at $298 \mathrm{~K}$ were compared.) Full geometry optimisations were performed first with the PM3 method and the obtained geometries were further optimised with $\mathrm{HF} / 6-31 \mathrm{G}^{*} / / \mathrm{HF} / 6-31 \mathrm{G}^{*}$ ab initio calculations. The optimum structures together with geometric data of the intramolecular hydrogen bonds are in Fig. 8.

It is to be seen that each close contact can be regarded as hydrogen bond, since the distances of the heavy atoms are within the sum of their van der Waals radii $(\mathrm{O} \cdots \mathrm{N}<307$ $\mathrm{pm})$ and the hydrogen points towards the acceptor. ${ }^{20,21}$ The lengths of the hydrogen bonds mirror the experimental finding

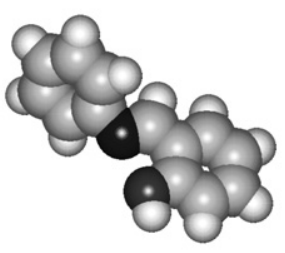

Bout

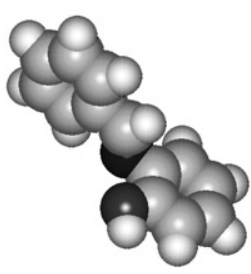

Cout

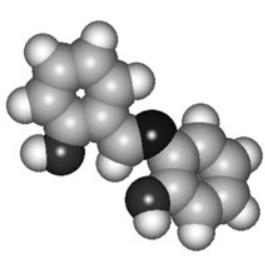

DCoutNout

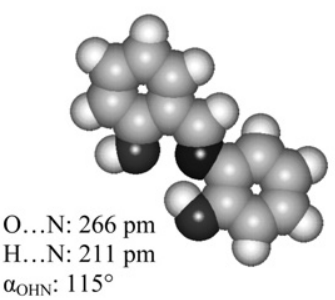

DCoutNin

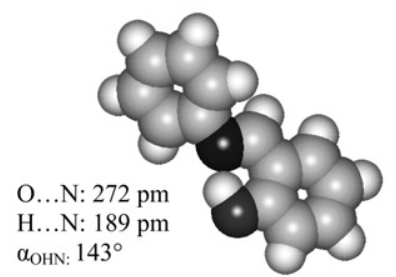

Bin

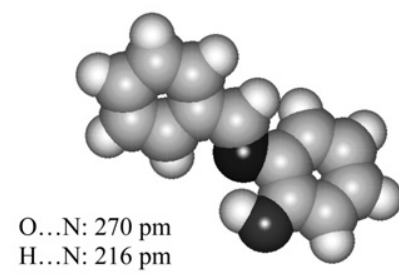
$\alpha_{\mathrm{OHN}}: 114^{\circ}$

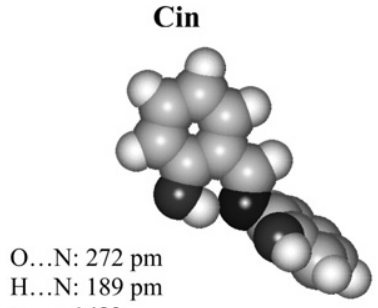

$\alpha_{\mathrm{OHN}}: 142^{\circ}$

DCinNout

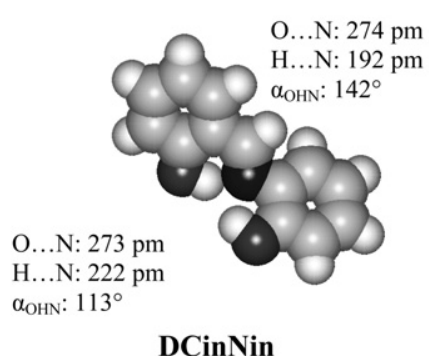

DCinNin
Table 1 Energy gain due to intramolecular hydrogen bond(s)

\begin{tabular}{llc}
\hline & \multicolumn{2}{l}{ Energy difference $/ \mathrm{kcal} \mathrm{mol}^{-1}$} \\
\cline { 2 - 3 } & PM3 & $\mathrm{HF} / 6-31 \mathrm{G}^{*} / / 6-31 \mathrm{G}^{*}$ \\
\hline$E_{\mathbf{B i n}}-E_{\mathbf{B o u t}}$ & -5.03 & -11.93 \\
$E_{\mathbf{C i n}}-E_{\mathbf{C o u t}}$ & -2.42 & -6.85 \\
$E_{\mathbf{D C i n N o u t}}-E_{\mathbf{D C o u t N o u t}}$ & -5.22 & -6.11 \\
$E_{\mathbf{D C o u t N i n}}-E_{\text {DCoutNout }}$ & -2.86 & -2.35 \\
$E_{\mathbf{D C i n N i n}}-E_{\mathbf{D C o u t N o u t}}$ & -7.26 & -10.80 \\
\hline
\end{tabular}

that the quasi six-membered ring is geometrically more favourable for hydrogen bond formation (molecules Bin and DCinNout) than the quasi five-membered ring (molecules $\mathbf{C i n}$ and DCoutNin).

Hydrogen bond formation results in stabilisation as reflected by both the semiempirical and the $a b$ initio calculations (Table 1). Generally, $a b$ initio calculations reported higher stabilisation energies than the PM3 computations.

Stabilisation is appreciable upon the formation of the quasisix-membered rings and moderate upon that of the quasi fivemembered ones. Interestingly, the energy gain for DCinNin was found to be higher than the sum the energy gains obtained for DCinNout and DCoutNin. Consequently, the monomer with as many close contacts as possible should be the most abundant species. Measurements did not fully reflect this statement, but it is clear that monomeric hydrogen bonded species of various kinds overwhelm that not having intermolecular hydrogen bonds at all (DCoutNout).

Although the five and especially the six-membered rings were stabilised by intramolecular hydrogen bonds, in these molecules, as experimental results obtained in solution indicated, complete hydrogen transfer, i.e. the a formation of a quinoidal structure was not found.

For molecule D, however, both intra- and intermolecular hydrogen bonding interactions were identified occurring via a quinoidal structure. Molecular modeling revealed that intermolecular hydrogen bond could form between the oxygen of the quinoidal carbonyl group of one molecule with the proton of the $\mathrm{OH}$ group of the other. Two such interactions are viable. Intramolecular hydrogen bond can be formed between the carbonyl oxygen and proton of the $\mathrm{C}-\mathrm{H}$ bond in the bridge connecting the two rings. Once again two such interactions can exist. The ab initio optimised dimer together with geometric data is depicted in Fig. 9.

$A b$ initio computations revealed that tautomerisation was not favoured: the related compound (DCoutNin) was $2.97 \mathrm{kcal}$ $\mathrm{mol}^{-1}$ more stable than the monomeric quinoidal tautomer. That has to be the reason why the tautomeric forms of any molecules investigated was not found by IR spectroscopy in solution. Even in the solid state only compound D showed signs of tautomer formation. By computation $21.3 \mathrm{kcal} \mathrm{mol}^{-1}$ stabilisation energy was obtained due to dimerisation, i.e. the

Fig. 8 Optimised geometries with geometric data of the intramolecular hydrogen bonds $\left(\mathrm{HF} / 6-31 \mathrm{G}^{*} / / \mathrm{HF} / 6-31 \mathrm{G}^{*}\right.$ theoretical level) for the various conformers of molecules $\mathbf{B}, \mathbf{C}$ and $\mathbf{D}$.

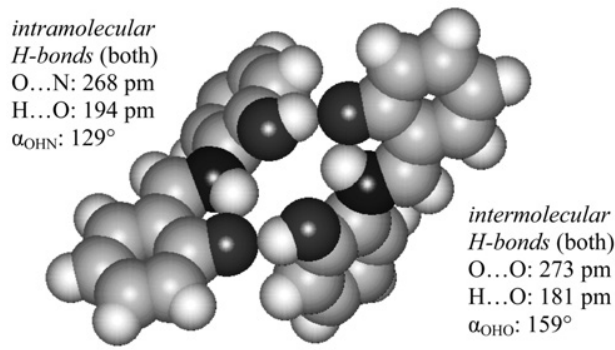

Fig. 9 The optimised geometry with geometric data of the inter- and the intramolecular hydrogen bonds $\left(\mathrm{HF} / 6-31 \mathrm{G}^{*} / / \mathrm{HF} / 6-31 \mathrm{G}^{*}\right.$ theoretical level) of the quinoidal dimer of molecule $\mathbf{D}$. 
formation of two intermolecular hydrogen bonds. The energy gained by the hydrogen bonds afforded the energy necessary for tautomerisation, comfortably.

Although detailed theoretical analysis of the normal modes obtainable from the force matrices is not the intention of this study the typical vibrations $(-\mathrm{N}=\mathrm{C},-\mathrm{OH}$ stretching modes) were identified and compared to the experimentally observed ones. Generally, force matrices of PM3 calculations could not reproduce these vibrations well, however, with the $\mathrm{HF} /$ 6-31G*//HF/6-31 $\mathrm{G}^{*}$ method more success was achieved, when a scaling factor of 0.8953 was used..$^{22}$ Experimental data for the $-\mathrm{N}=\mathrm{C}$ stretching mode for the compounds dissolved in acetone- $\mathrm{d}_{6}$ are $1629 \mathrm{~cm}^{-1}, 1620 \mathrm{~cm}^{-1}, 1626 \mathrm{~cm}^{-1}, 1619 \mathrm{~cm}^{-1}$ respectively, while the computed data for the base molecule and the hydrogen bond stabilised monomers (A, Bin, Cin, DCinNin) are $1656 \mathrm{~cm}^{-1}, 1631 \mathrm{~cm}^{-1}, 1643 \mathrm{~cm}^{-1}, 1625$ $\mathrm{cm}^{-1}$, respectively. It is to be seen that the relevant pairs are acceptably close to each other and the sequence are also reproduced well, indicating where possible intramolecular hydrogen bonds stabilise the structures. As concerns the $-\mathrm{OH}$ stretching mode, for Bin it was found at $3200 \mathrm{~cm}^{-1}$ indicating strongly associated $\mathrm{OH}$, for Cin at $3450 \mathrm{~cm}^{-1}$ pointing at weakly associated $\mathrm{OH}$. Both observations compare acceptably to the experimentally found $3290 \mathrm{~cm}^{-1}$ and $3415 \mathrm{~cm}^{-1}$, respectively. For compound $\mathbf{D}$ both types were identified experimentally (in solution) as well as computationally. The quinoidal dimer gives computationally $1643 \mathrm{~cm}^{-1}$ for the $-\mathrm{C}=\mathrm{O}$ stretch, while the experimental value is $1636 \mathrm{~cm}^{-1}$, a convincing coincidence.

\section{Conclusions}

On the basis of experimental observations and molecular modeling calculations it can be concluded that intramolecular hydrogen bonding prescribes the geometry of molecules $\mathbf{B}$ and $\mathbf{C}$ in the solid state as well as in solution.A quasi six-membered ring is formed for molecule $\mathbf{B}$ and a five-membered one for molecule C. Spectroscopic measurements indicated and computations quantified that the hydrogen bonding is stronger for the former molecule than that for the latter. Spectroscopic results show that both aromatic rings retain their aromaticity, i.e., no charge transfer resulting in the formation of quinoidal structure was observed. For compound D dimerization takes place in the solid state with the appearance of intermolecular hydrogen bonds keeping intramolecular hydrogen bondings as well. At the same time the benzylidene ring loses its aromaticity and the structure becomes quinoidal. In aprotic solutions, however, dimerisation does not occur, nor the loss of aromaticity takes place, the structure of compound $\mathbf{D}$ is determined by intramolecular hydrogen bonds.

\section{Acknowledgements}

Financial support for this work provided by the Ministry of Education via grant FKFP 0286/2000 is highly appreciated.

\section{References}

1 M. Smith and J. March, March's Advanced Organic Chemistry, Wiley, New York, 2001.

2 D. Voet and J. G. Voet, Biochemistry, Wiley, New York, 1995; T. M. Devlin, Textbook of Biochemistry with Clinical Correlations, John Wiley, New York, 2001

3 N. Hoshino, T. Inabe, T. Mitani and Y. Maruyama, Bull. Chem Soc. Jpn, 1988, 61, 4207.

4 M. D. Cohen and G. M. J. Scmidt, J. Phys. Chem., 1962, 66, 2442.

5 A. W. Baker and A. T. Shulgin, J. Am. Chem. Soc., 1959, 81, 1523.

6 S. V. Lindeman, M. Yu. Antipin and Yu. T. Struchkov, Kristallografiya, 1988, 33, 365.

7 J. Császár and M. Bizony, Magy. Kém. Foly., 1997, 103, 29.

8 J. W. Ledbetter, J. Phys. Chem., 1977, 81, 54.

9 HyperChem 7.0, Hypercube, Inc., Gainesville, FL, 2002.

10 J. J. P. Stewart, J. Comput. Chem., 1989, 10, 209; J. J. P. Stewart, J. Comput. Chem., 1989, 10, 221.

11 B. Ostrowska and A. Tramer, Acta Phys. Pol., 1967, 33, 111.

12 Z. Meic and G. Baranovic, Pure Appl. Chem., 1989, 61, 2129.

13 V. I. Minkin, O. A. Osipov and V. N. Sheinker, Zh. Fiz. Khim., 1970, 44, 23

14 J. W. Ledbetter, J. Phys. Chem., 1982, 86, 2449.

15 T. Inabe, T. Mitani, Y. Maruyama and S. Takeda, Bull. Chem. Soc. Jpn, 1994, 67, 612.

16 K. Wozniak, H. He, J. Klinowski, W. Jones, T. Dziembowska and E. Grech, J. Chem. Soc., Faraday Trans., 1995, 91, 77.

17 A. G. J. Ligtenbarg, R. Hage, A. Meetsma and B. L. Feringa, J. Chem. Soc., Perkin Trans. 2, 1999, 807.

18 Z. Rozwadowski, E. Majewski, T. Dziembowska and P. E Hansen, J. Chem. Soc., Perkin Trans. 2, 1999, 2809.

19 A. Filarowski, A. Koll and T. Glowiak, J. Chem. Soc., Perkin Trans. 2, 2002, 835 .

20 A. Bondi, J. Phys. Chem., 1964, 68, 441.

21 C. B. Aakeroy, T. A. Evans, K. R. Seddon and I. Pálinkó, New J. Chem., 1999, 23, 145.

22 A. P. Scott and L. Radom, J. Phys. Chem., 1996, 100, 16502. 